\title{
Sistema de Manufatura Inteligente para Seleção de Mangas utilizando Deep Learning
}

\author{
Carine R. de Almeida Gottschall* \\ João Erivando S. Marques * Cleia S. Libarino* \\ José A. Diaz Amado* \\ * Departamento de Engenharia Elétrica, Instituto Federal da Bahia, BA \\ (e-mails: carineragottschall@gmail.com, joaoerivandosm@gmail.com, \\ libarino.cleia@gmail.com,sportingjada1@hotmail.com).
}

\begin{abstract}
This work intends to develop an intelligent manufacturing system able to classify and select mangoes according to their degree of maturation, using deep learning networks, specifically convolutional, allied to a process of computer vision. For this, a database was constructed using images of mangoes Tommy Atkins, Haden and Palmer, in three maturing stages distinct, green, mature and very mature. The manufactured system has a conveyor belt for transporting the fruit, a support for positioning the devices and three ramps for steering. The performance of the neural network was evaluated through the analysis of the accuracy and losses in the validation tests. The results presented showed an accuracy higher than $99 \%$ in the intelligent selection process.

Resumo: Este trabalho possui o intuito de desenvolver um sistema de manufatura inteligente capaz de classificar e selecionar mangas de acordo com o seu grau de maturação, utilizando redes deep learning, especificamente convolucionais, aliada a um processo de visão computacional . Para isso, foi construída uma base de dados utilizando imagens de mangas Tommy Atkins, Haden e Palmer, em três estágios de maturação distintos, verde, maduro e muito maduro. O sistema manufaturado dispõe de uma esteira para transporte do fruto, um suporte para posicionamento dos dispositivos e três rampas de direcionamento. O desempenho da rede neural foi avaliado mediante a análise da acurácia e perdas nos testes de validação . Os resultados apresentados mostraram uma acurácia superior aos $99 \%$ no processo de seleção inteligente.
\end{abstract}

Keywords: Deep Learning; Convolutional Neural Networks; Computational Vision; Manufacturing System; RGB Images; Mangoes.

Palavras-chaves: Aprendizado Profundo; Redes Neurais Convolucionais; Visão Computacional; Sistema de Manufatura; Imagens RGB; Mangas.

\section{INTRODUÇÃO}

O avanço constante na computação e na robótica, decorrido maiormente após o ano 2000, viabilizou a execução de atividades noutro tempo impraticáveis e que favorecem as mais diversas áreas da ciência. Um campo de grande difusão da robótica e automação foi a agricultura. De acordo com o levantamento da Comissão Brasileira de Agricultura de Precisão (CBAP), 67\% das propriedades agrícolas do país empregam algum tipo de tecnologia, seja na gestão dos negócios ou nas atividades de cultivo e colheita da produção (Brasil, 2017). No entanto, ainda há demanda por métodos mais eficientes, não somente no monitoramento da fase de cultivo, mas também, na inspeção e seleção dos produtos pós-colheita, para obterse o melhor aproveitamento da produção.

O Brasil é um dos maiores produtores frutícolas, com destaque para as mangas, ocupando o $6^{\circ}$ lugar no ranking de maiores produtores de mangas da Organização das Nações Unidas para Alimentação e Agricultura (FAO) em 2014 Comércio Exterior (COMEX, 2017), em contraposição a isso tem-se a recepção dos frutos pelo consumidor, que é afetada por grandes perdas ocorridas principalmente no período pós-colheita. Há ainda uma meta estipulada pela Organização das Nações Unidas (ONU) através do Objetivo de Desenvolvimento Sustentável (ODS) que prevê, dentre outras medidas, a redução das perdas nos sistemas de produção e abastecimento incluindo o momento póscolheita até 2030. Diante disso, há necessidade da incorporação de processos mais eficientes aplicados à seleção e classificação de mangas, afinal, são esses procedimentos que definem se determinado fruto está ou não com a qualidade exigida pelo consumidor. Um importante indicador da qualidade da manga é a cor de sua casca, como analisado pela Empresa Brasileira de Pesquisa Agropecuária (EMBRAPA, 2000).

No caso da avaliação do estágio de maturação, os padrões são definidos em níveis, os quais indicam a aparência da fruta em uma dada etapa de seu desenvolvimento, como este processo compreende um fenômeno não linear e ainda modificável pelas condições ambientais e sendo a imagem da fruta também influenciada por estes fatores, uma vez que reproduz as condições da superfície dos frutos, se torna viável a utilização de imagens para determinar o 
estágio de maturação dos mesmos, como aponta Carvalho et al. (2014). Eles apresentaram um método baseado em inspeção visual automática para a classificação de mangas da variedade Tommy Atkins através da avaliação da cor da casca aplicando Machine Learning (ML). Os autores desenvolveram um método de classificação automático baseado na conversão de escala de cores Hue Saturation Light (HSL) com a descrição do percentual de cada componente da escala em histograma e a associação do valor do nível para cada percentual de cores. O histograma da imagem capturada da fruta era comparado ao histograma médio encontrado no passo anterior para rotular a mesma. Um outro sistema de classificação de maturação de mangas utilizando ML foi desenvolvido por Silva et al. (2017), o qual aplicou Redes Neurais Artificiais (RNA) para realizar o processo de aprendizagem. O sistema permitia a distinção de três classes através da captura e pré-processamento das imagens para obtenção das porcentagens de cada componente da escala de cores Red Green Blue (RGB). Os trabalhos de classificação de frutas mais recentes, Sa et al. (2016), Puttemans et al. (2016), Bargoti e Underwood (2017), Muresan e Oltean (2018) e (Bath et al. 2018), empregam técnicas inteligente que permitem a análise do fruto pelo próprio algoritmo de aprendizagem sem a necessidade de um pré-processamento para extração das caracterísiticas a serem avaliadas. Isso é possibilitado mediante o emprego das Convolutional Neural Networks (CNN), que consistem em um método de aprendizagem profunda projetado para trabalhar com imagens (Lecun et al. 2001).

O trabalho desenvolvido por Sa et al. (2016) é aplicado a colheita robótica e tem como propósito identificar frutas em pomares, incluindo pimenta doce, melão, maçã, abacate, manga e laranja. O sistema utiliza a fusão do modelo RGB e Near Infra Red (NIR) e um algoritmo VGG-16 para treinamento, além adaptar uma rede convolucional bem mais rápida conhecida como Faster R-CNN que permitiu obter um desempenho superior às redes CNN já existentes e a identificação do posicionamento dos frutos, mesmo com a existência de planos de fundos barulhentos. Além disso, também utilizam uma técnica de transferência de aprendizdo entre pomares, facilitando o processo de treinamento da rede.

Bargoti e Underwood (2017) apresentam uma proposta e abordagem semelhante aos autores anteriores para frutas do tipo manga, amêndoa e maçã, além do método de transferência de aprendizagem eles também empregam uma técnica de aumento de dados, resultando em uma redução maior que duas vezes no número de imagens de treinamento necessárias. Diante das análises feitas, os autores observam que um meio menos dispendioso de reduzir o erro de rotulagem seria usar a saída do detector treinado para limpar os dados de fundo da imagem com um ser humano no circuito.

Já Muresan e Oltean (2018) utilizaram uma rede neural convolutivas para classificação de 95 tipos de frutas distintas, com base de dados própria contendo imagens de frutas rotacionadas em 360 graus. As imagens eram préprocessadas para retirada do fundo ruidoso, de modo a reproduzir apenas o fruto. A arquitetura empregada foi implementada pelos próprios autores que também aplicaram técnicas de aumento de dados através da variação de matiz e saturação e inversão vertical e horizontal das imagens empregadas no treinamento do algoritmo, obtendo bom desempenho no que diz respeito a acurácia e perda tanto nas fases de treinamento como de teste.

O estado da arte ligado a esta temática apresenta diversas técnicas de aprendizagem de máquina aplicados a classificação e seleção de frutas. As técnicas que aprendizado profundo apresentam melhor desempenho tanto na classificação em si, quanto no tempo de processamento do sistema. No entanto, estes não propõem a avaliação da maturação das mangas aplicando redes CNN e sua seleção na etapa pós-colheita. Em vista disso, a contribuição deste trabalho está na implementação de um processo de seleção pós-colheita de mangas quanto ao seu grau de maturação, em: verdes, maduras ou muito maduras, utilizando deep learning por meio de uma rede $\mathrm{CNN}$, aplicado a um sistema de manufatura automatizado. O artigo se encontra organizado da seguinte maneira: A Seção 2 apresenta os materiais e métodos empregados no desenvolvimento do sistema. A Seção 3 apresenta os resultados obtidos. As conclusões do trabalho são apresentadas na Seção 4.

\section{MATERIAIS E MÉTODOS}

O sistema foi construído e organizado em três blocos distintos: o sistema de visão computacional, o sistema de classificação e o sistema de seleção do fruto. Estes comunicam entre si de tal forma que o sistema de visão é responsável por capturar informações do processo, as imagens das mangas, que são aplicadas como entradas no sistema de classificação, o qual rotula o fruto de acordo a sua maturação. Por fim, esse resultado é utilizado para o controle de acionamento do sistema de seleção, a Figura 1 retrata o diagrama de funcionamento do sistema.

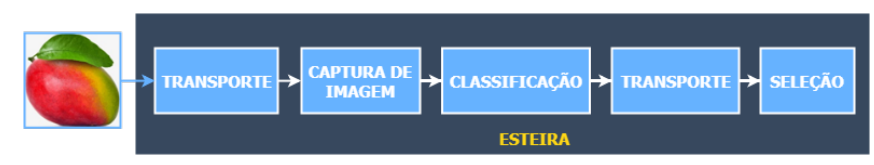

Figura 1. Diagrama esquemático de funcionamento do sistema inteligente.

\subsection{Sistema de Visão Computacional}

O sistema de visão computacional é composto por uma câmera que permite o sensoriamento e captura de características visuais do objeto de trabalho. Para a captura de imagens foi necessário um controle de iluminação garantindo um bom índice de reprodução de cor (IRC), o qual quantifica a fidelidade com que as cores de um objeto são reproduzidas sob determinada fonte de luz artificial. Assim utilizou-se um suporte simulando uma câmara escura sobre o qual foram dispostas quatro lâmpadas incadescentes, com IRC próximo a 100, nas extremidades do teto, eliminando o máximo possível a influência da iluminação ambiente, reduzindo a formação de sombras e a proporcionando a iluminação de toda a superfície do fruto.

Tanto o sistema de visão quanto o sistema de classificação exigem um computador para processar os dados. Optou-se por empregar o Raspberry Pi 3, por se tratar de um System on Chip (SoC), possuir compatibilidade com pacotes e frameworks necessários à aquisição e processamento de imagens e à execução dos algoritmos de rede, além de ser 
de fácil acoplamento a estrutura física do sistema.

As imagens das frutas foram coletadas através do módulo Raspberry Pi Camera modelo RP_ov5647, pelo fato deste possuir desempenho adequado à aplicação desejada e ser compatível com o microcomputador e o sistema operacional instalado. A câmera foi acoplada através do cabo flat, diretamente à placa pela Camera Interface Serial (CSI) e posicionada no centro do teto inferior do suporte. A Figura 2 mostra a estrutura do suporte e o posicionamento da câmera e do sistema de iluminação sobre o mesmo.

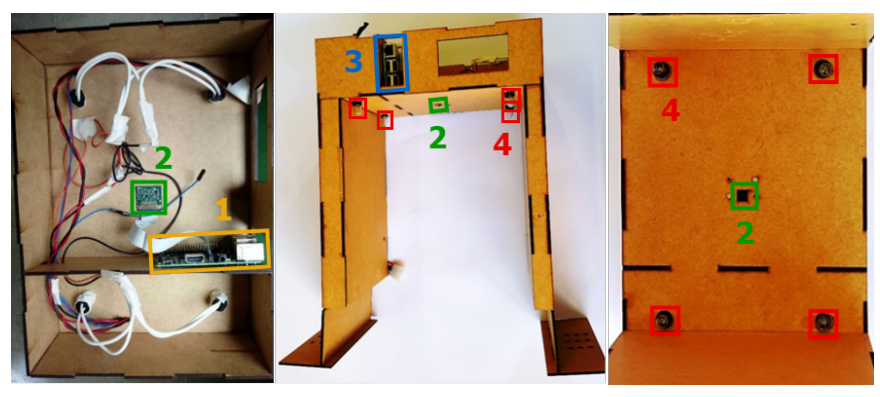

Figura 2. Suporte e disposição do sistema de visão computacional e iluminação: (1) Raspberry Pi 3; (2) Raspberry Pi Camera; (3) Acesso aos periféricos do Rapberry Pi; (4) Lâmpadas incandescentes.

\subsection{Sistema de Classificação de Mangas}

A implementação do sistema de classificação de mangas decorreu com a definção da topologia de rede a ser empregue para categorizar a maturação das mangas, seguida da construção da base de dados, Dataset, do treinamento do modelo e dos testes de validação, os quais serão discorridos nas próximas subseções.

Implementação da rede neural convolutiva A arquitetura escolhida foi a construída por Muresan e Oltean (2018), pelo fato de possuir ótima performance e acurácia na classificação de 95 classes de frutas distintas. Outro ponto favorável do modelo é sua topologia, composta por apenas quatro camadas convolucionais que são capazes de extrair importantes características de padrões de coloração e dimensão das frutas e apresentam uma técnica de aumento de dados para expandir a quantidade de imagens de treinamento através da inversão vertical e horizontal das mesmas, melhorando o processo de treinamento. A rede recebe como entrada uma imagem com 100 pixels de altura e 100 pixels de largura com um formato .jpg e quatro camadas de profundidade. A profundidade da camada de entrada é proveniente de um pré-processamento da imagem RBG capturada pela câmera com 3 camadas de profundidade, que é filtrada para o sistema Hue Saturation Value (HSV) e escala de cinza, resultando em uma imagem HSV com três camadas de profundidade e uma imagem em escala de cinza com uma camada de profundidade, como mostra a Figura 3.

Construção da base de dados As imagens para treinamento e teste da rede foram capturadas através da Picamera e obtidas cerca de 40 fotos para 30 mangas diferentes, sendo elas verdes, maduras ou muito maduras, com a disposição de uma única fruta por vez sobre a esteira e com o sistema de iluminação acionado. As mangas utilizadas no

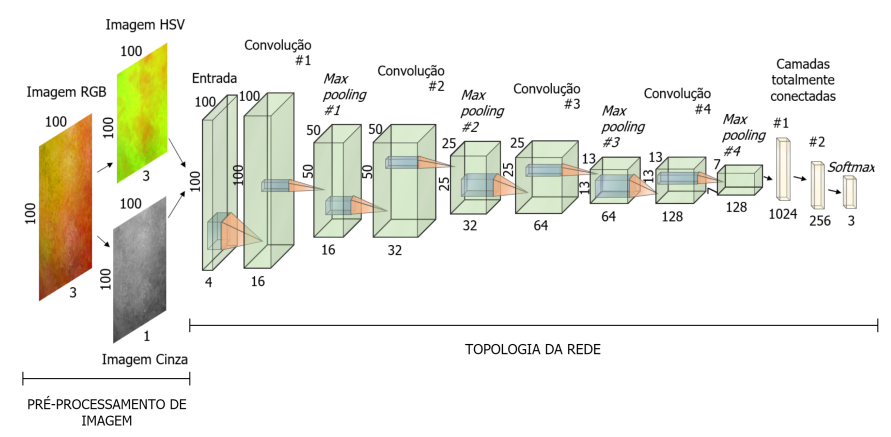

Figura 3. Arquitetura da rede neural convolutiva utilizada. Fonte: Adaptado de Muresan e Oltean (2018, p. 18).

Dataset foram do tipo Tommy Atkins, Haden ou Adam e Palmer, pois as mesmas apresentam características de cores semelhantes durante a maturação. Tanto para a coleta de dados quanto para a análise da manga, foi definido um único lado do fruto, visto que ao longo da sua superfície as tonalidades são variantes. A metodologia adotada para rotular as frutas foi baseada no padrão de coloração de estágios de maturação da Embrapa (Assis e Lima, 2008). O estágio 1, visto na Figura 4, corresponde ao rótulo verde, o estágio 2 ao rótulo madura e o estágio 3 ao muito madura. A dimensão da captura das imagens foi de 1000 pixels

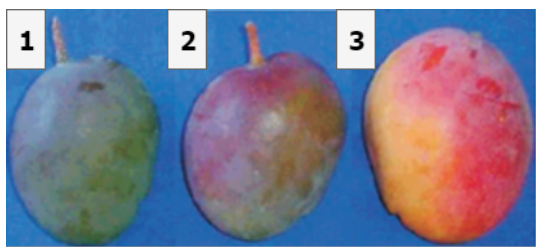

Figura 4. Escala de maturação da manga Tommy Atkins segundo a coloração da casca.

Fonte: Adaptado de Assis e Lima (2008).

de altura e 1000 pixels de largura. Para acrescentar à quantidade de imagens do Dataset e à variação dos padrões para cada estágio foram extraídas fotografias de mangas através do aplicativo Google Imagens, as quais ajustadas, no que diz respeito ao brilho, saturação, matiz e cor da imagem para melhor adequação aos padrões definidos. As imagens obtidas foram recortadas de modo a compreender apenas padrões de coloração do fruto sem contemplar sua forma, já que essa é invariante durante o processo de maturação do fruto. Posteriormente, redimensionou-se as imagens para 100x100 como mostrado na Figura 5 com representação de cores RGB.

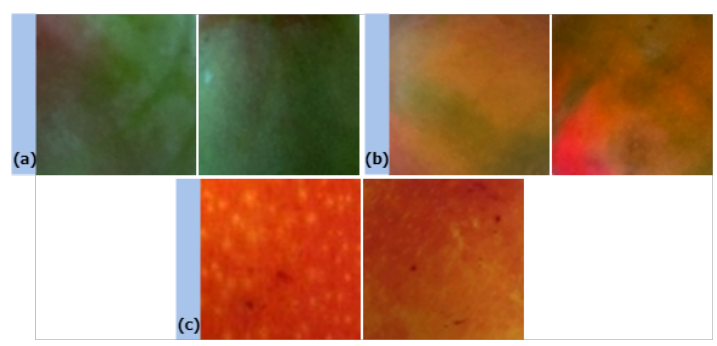

Figura 5. Amostra de imagens 100x100 utilizadas para treinamento e validação de rede: (a) mangas verdes; (b) mangas maduras; (c) mangas muito maduras. 
Treinamento da rede neural No treinamento da rede neural empregou-se 3.598 imagens rotuladas, que após serem submetidas as técnicas de aumento de dados, teve sua quantidade triplicada para 10.794 imagens. Foram realizados quatro treinamentos nos quais aplicou-se uma taxa de aprendizado, learning rate, variante, para evitar o overfitting, impedindo que o modelo se ajuste aparentemente muito bem ao conjunto de dados observado, mas se mostre ineficaz para prever novos resultados. A taxa inicial foi de 0,001 e a final de 0,00001. Os treinamentos foram executados mediante um computador laptop com um processador CPU intel-core i7 e sistema operacional Windows 10. Foram utilizadas diferentes combinações de parâmetros, variando a quantidade de épocas e imagens no mini-lote, no intuito de dispor da melhor delas no processo de classificação. O modelo foi treinado para parada através de um número de épocas definido.

Avaliação do desempenho da rede neural Para avaliar o desempenho da rede foram adotados de quatro parâmetros: a perda e a acurácia do treinamento; a resposta da rede em relação à categoria de maturação predefinida para os dados de validação; e o teste prático, no qual a rede neural foi implementada no sistema e a esteira foi acionada, iniciando o transporte de mangas cuja maturação já era conhecida pelo operador, e posteriormente comparando com a maturação indicada pela predição da rede. A realização dos testes consistiu na restauração dos pesos treinados para imagens desconhecidas pela rede, ou seja, que não haviam sido utilizadas na etapa de treinamento. A quantidade de imagens empregadas para teste foi definida através de uma proporção de $70 \%$ das imagens da base de dados para a fase treinamento, $20 \%$ para testes de validação e $10 \%$ para testes individuais, a Tabela 1 apresenta a quantidade de imagens por rótulo e por etapa.

Tabela 1. Número de imagens para cada rótulo empregada em cada etapa.

\begin{tabular}{l|c|c|c}
\hline Rótulo & $\begin{array}{c}\mathbf{N}^{\circ} \text { imagens } \\
\text { treinamento }\end{array}$ & $\begin{array}{c}\mathbf{N}^{\mathbf{}} \\
\text { imagens } \\
\text { teste }\end{array}$ & $\begin{array}{c}\mathbf{N}^{\mathbf{0}} \text { imagens } \\
\text { testes } \\
\text { individuais }\end{array}$ \\
\hline Verde & 1.200 & 340 & 170 \\
\hline Madura & 1.195 & 340 & 170 \\
\hline Muito madura & 1.203 & 340 & 170 \\
\hline
\end{tabular}

\subsection{Seleção do Fruto}

O processo de seleção inicia-se após a predição da maturação da manga, a qual é transportada até o atuador correspondente para ser seja direcionada ao caminho adequado. Assim, foi implementado um controle de velocidade e acionamento da esteira, bem como dos atuadores de seleção. A movimentação da esteira procedeu-se mediante o acionamento de um motor DC de $12 \mathrm{~V}$, com um único eixo de tracionado. Para tanto aplicou-se um gerador de sinal Pulse Width Modulation (PWM) e uma chave ON/OFF com o fim de permitir a parada do processo e a permanência do fruto no local adequado, centralizado com a câmera, para captura da imagem e, logo após o processo de classificação, o religamento do motor, para o transporte da fruta sob a esteira até chegar ao local de seleção.
Foram elaborados selecionadores retangulares com comprimento curvo de plástico com $5 \mathrm{~mm}$ de espessura, $26 \mathrm{~cm}$ de comprimento e $5 \mathrm{~cm}$ de largura. Como o sistema distingue três classes de mangas, implantou-se dois selecionadores rotativos na estrutura, acoplados cada um ao eixo de um motor diferente, enquanto que, a terceira classe do fruto é transportada até o final da esteira e rola por uma rampa, como mostra a Figura 6. A movimentação dos selecio-

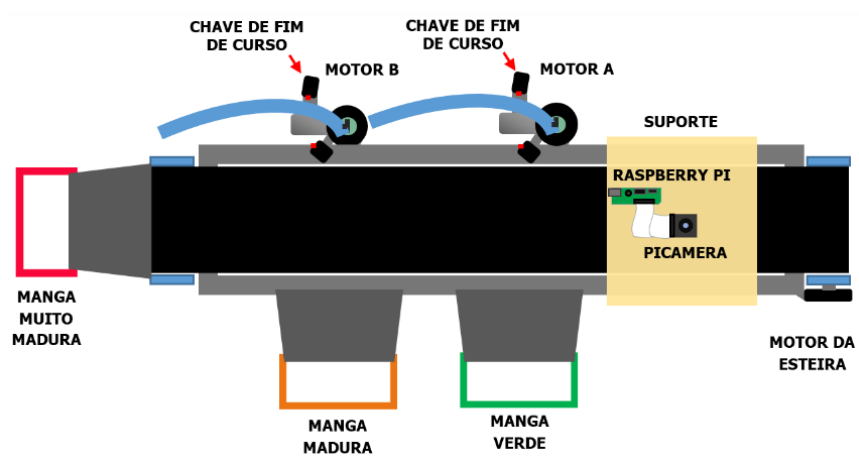

Figura 6. Diagrama esquemático do sistema de seleção e classificação com vista superior.

nadores se deu através de dois motores mabuchi Direct Current (DC) de vidro elétrico de $12 \mathrm{~V}$ de lado direito com oito dentes e consumo de 1,3 A. O acoplamento com o eixo dos motores estabeleceu-se por meio de uma peça adaptativa de durepoxi, a qual, por sua vez, foi parafusada ao braço de plástico. Para a inversão de rotação dos dois motores empregou-se um módulo Ponte H L298N, com uma alimentação de $12 \mathrm{~V}$ e para o controle de velocidade utilizou-se as saídas PWM da placa Arduino. O mecanismo de seleção implantado foi baseado na retenção do fruto após a classificação, sendo ele verde ou maduro.

Para marcar o início e o fim de curso que delimita o movimento dos selecionadores na estrutura da manufatura, foram utilizadas chaves de fim de curso. Cada motor possui duas chaves de fim de curso, estrategicamente posicionadas como visto na Figur 6, que marcam o início e o fim de curso do motor e quando acionadas realizam a parada do motor no ciclo em que o mesmo se encontra. As frutas retidas pelos selecionadores continuam submetidas a velocidade do motor da esteira e devido ao ângulo e o formato do selecionador, como pode ser visto no diagrama da Figura 6 , rola até cair pela rampa correspondente a sua classe. Caso a manga esteja muito madura ela é transportada até o final da esteira e desce por uma terceira rampa.

\section{RESULTADOS E DISCUSSÕES}

Foram executados vários cenários de treinamento da rede neural utilizando diferentes quantidade de imagens de mini-lote e de épocas. A cada 50 épocas ou passos, foi calculada a precisão através do método de cross validation ou entropia cruzada. Os resultados finais de acurácia e perda correspondente a cada cenário são apresentados na Tabela 2. O tempo gasto no treinamento para configurações com uma quantidade maior de imagens de mini-lote é superior ao tempo gasto empregando mini-lotes menores, isso ocorre devido ao tipo de processador e a alocação de memória realizada pelo mesmo durante o processo. Como a CPU deve dividir as tarefas do seu processador para 
Tabela 2. Resultado do treinamento executado para diferentes configurações de parâmetros da rede.

\begin{tabular}{|c|c|c|c|c|}
\hline \multicolumn{3}{|c|}{ Treinamento } & Acurácia & Perda \\
\hline \multirow{3}{*}{$\mathbf{T 1}$} & Mini-lote & 60 & \multirow{3}{*}{$99,00 \%$} & \multirow{3}{*}{$4,24 \%$} \\
\hline & Épocas & 10000 & & \\
\hline & Duração & $12 \mathrm{hrs} 32 \mathrm{~min}$ & & \\
\hline \multirow{3}{*}{ T2 } & Mini-lote & 40 & \multirow{3}{*}{$99,14 \%$} & \multirow{3}{*}{$5,34 \%$} \\
\hline & Épocas & 8000 & & \\
\hline & Duração & 6hrs 33min & & \\
\hline \multirow{3}{*}{ T3 } & Mini-lote & 70 & \multirow{3}{*}{$99,08 \%$} & \multirow{3}{*}{$5,24 \%$} \\
\hline & Épocas & 7000 & & \\
\hline & Duração & 7hrs 53min & & \\
\hline \multirow{3}{*}{$\mathbf{T} 4$} & Mini-lote & 80 & \multirow{3}{*}{$99,58 \%$} & \multirow{3}{*}{$3,59 \%$} \\
\hline & Épocas & 8500 & & \\
\hline & Duração & 10hrs $07 \mathrm{~min}$ & & \\
\hline
\end{tabular}

manter outras funcionalidades do computador, ou seja, não possui dedicação exclusiva ao treinamento do modelo e sendo as imagens do pacote de mini-lote agrupadas uma por uma na memória RAM, é exigido um tempo maior para que o processador seja capaz de alocar todas as imagens para o treinamento. Assim, a execução de uma época do algoritmo de rede é muito mais dispendiosa para a máquina no que diz respeito ao tempo de execução.

Ao comparar o resultado do treinamento T1 com o T2, é visto que, apesar do segundo apresentar uma acurácia maior, ou seja, maior proporção de predições corretas em relação ao tamanho do conjunto de dados, a perda na proximidade das distribuições de probabilidade retornada pela rede em relação a distribuição verdadeira é maior para T2. Dessa forma, a configuração que melhor contempla a base de dados obtida foi em T4, aplicando um número de mini-lote de 80 imagens e 8500 passos, pois apresenta uma acurácia superior às outras, além de uma menor perda na distribuição de probabilidade das classes. Nesse cenário uma maior quantidade de informações é processada por lote, assim a rede pode aprender vários recursos para classificar as imagens.

Após a avaliação de desempenho do treinamento da rede, foram feitos testes com a finalidade de validá-los. Para isso, restaurou-se os arquivos com os pesos de cada configuração distinta treinada e aplicou-se ao conjunto de testes, que é composto por 340 imagens de cada classe. Os resultados foram analisados segundo a acurácia, ou seja, a quantidade de predições corretas realizadas pela rede para cada cenário são apresentados na Tabela 3.

Tabela 3. Testes realizados para validar cada configuração treinada.

\begin{tabular}{c|l|c|c|c|c}
\cline { 3 - 6 } \multicolumn{2}{c|}{} & \multicolumn{4}{c}{ Classe } \\
\hline \multirow{2}{*}{ Teste } & Madura & $\begin{array}{c}\text { Muito } \\
\text { madura }\end{array}$ & Verde & TOTAL \\
\cline { 3 - 6 } & $\mathrm{N}^{\mathrm{O}}$ amostras & 340 & 340 & 340 & 1020 \\
\hline \multirow{2}{*}{$\mathbf{T} 2$} & $\mathrm{~N}^{\mathrm{a}}$ acertos & 339 & 332 & 340 & 1011 \\
\cline { 2 - 6 } & $\mathrm{N}^{\mathrm{a}}$ amostras & 340 & 340 & 340 & 1020 \\
\hline \multirow{2}{*}{$\mathbf{T} 3$} & $\mathrm{~N}^{\mathrm{a}}$ acertos & 333 & 337 & 337 & 1007 \\
\cline { 2 - 6 } & $\mathrm{N}^{\mathrm{O}}$ amostras & 340 & 340 & 340 & 1020 \\
\hline \multirow{2}{*}{ T4 } & $\mathrm{N}^{\mathrm{a}}$ acertos & 334 & 333 & 337 & 1004 \\
\cline { 2 - 6 } & $\mathrm{N}^{\mathrm{O}}$ amostras & 340 & 340 & 340 & 1020 \\
\hline
\end{tabular}

As configurações aplicadas em T2 e T3, apesar de apresentarem acurácia superior à T1 na etapa de treino, mostram

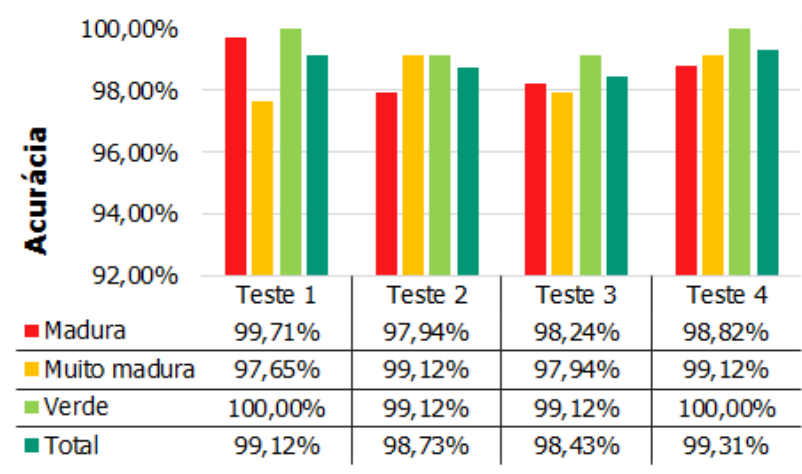

Figura 7. Acurácia resultante dos testes de validação para as quatro configurações de rede treinadas.

com desempenho inferior nos testes, principalmente no reconhecimento de mangas maduras e verdes, sendo a pior performance exibida por T3. Isso é resultado do overfitting do modelo para os dados de treinamento e da falta de generalização para outras imagens. Os parâmetros empregados em T1 exibem acurácia semelhante para as etapas de treino e teste, menor erro inerente as probabilidades no treinamento e predição correta para todas as mangas verdes no teste.

O padrão de coloração apresentado no estágio de maturação para frutas verdes possui a menor variação de característica diante dos demais estágios, o que refletiu nos resultados de teste, sendo essa classe a que exibe mais predições corretas diante todas as avaliadas. A quarta configuração foi a que apresentou desempenho superior tanto para o treinamento quanto para os testes, mostrando um desempenho de $100 \%$ para as predições de mangas verdes e uma acurácia total de 99,31\%, assim, optou-se por aplicá-lo para os testes práticos no sistema real de classificação.

Para verificar o grau de ligação que a análise dos dados de validação com a realidade, efetuou-se a implementação da rede já treinada no Raspberry Pi 3, juntamente com o software de controle do sistema, que realiza a coleta das imagens dos frutos, processamento e comunicação serial com o sistema de seleção. Foram utilizadas três mangas Palmer, uma para cada classe, mostradas na Figura 8, com a finalidade de averiguar o desempenho da rede para esse tipo de manga. Elas foram dispostas sobre a esteira sempre com o lado inferior voltado para câmera, com uma altura de $14 \mathrm{~cm}$ entre a câmera e a esteira. O sistema tem uma cadência de 12 segundos para coleta e classificação da imagem e um tempo de transporte e seleção de fruta de 5 segundos para as mangas verdes que são selecionadas pelo primeiro atuador e 7 segundos para as mangas maduras que acionam o segundo atuador, enquanto as frutas muito maduras levam 5 segundos para serem transportadas. As imagens capturadas, assim como a classificação e probabilidade das mesmas são apresentadas na Figura 9. Como exposto na Figura 9, a rede foi capaz de classificar corretamente os estágios de maturação para a manga Palmer. Observou-se que a predição retornada ainda é afetada pela posição que o operador insere o fruto na esteira, para que seja capturada toda a superfície do fruto, como exposto na primeira linha e quinta coluna da Figura 9, apesar de rotular corretamente a maturação da manga, a rede responde com uma probabilidade baixa inerente a ela. 


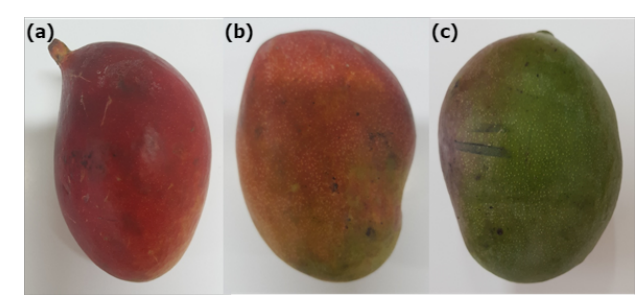

Figura 8. Mangas Palmer empregadas nos testes: (a) muito madura; (b) madura; (c) verde.

A imagem obtida compreende uma boa parte da esteira afetando a probabilidade da predição. Para as demais capturas, o sistema apresentou uma boa distribuição de probabilidade, ou seja, uma perda muito pequena. Nos

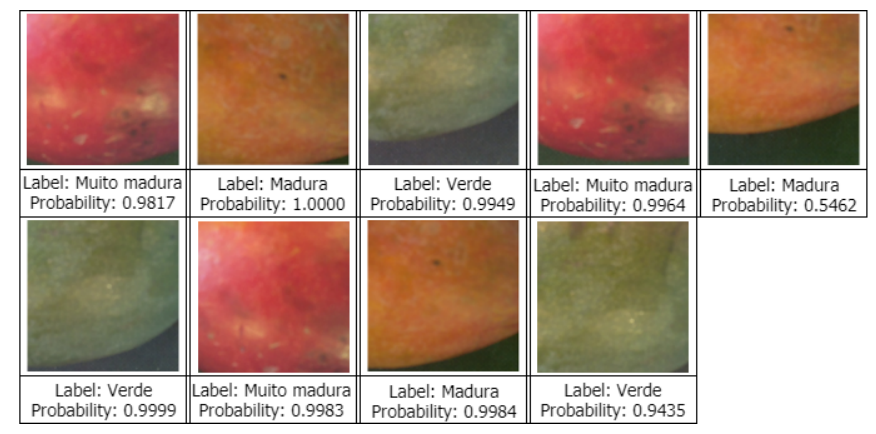

Figura 9. Imagens capturadas pelo sistema e a predição e probabilidade retornada pelo mesmo.

testes realizados, a comunicação serial entre o Raspberry Pi e Arduino se deu de maneira eficiente, sem perdas de informação. O acionamento dos atuadores ocorreu como esperado, o primeiro selecionador, conseguinte ao suporte, foi movimentado na presença de mangas maduras, enquanto o selecionador subsequente foi acionado para a presença de mangas verdes, e por fim, nenhum selecionador foi acionado para as mangas muito maduras, que seguiram até o final da esteira, rolando pela terceira rampa. O protótipo referente ao sistema de manufatura desenvolvido se encontra na Figura 10.

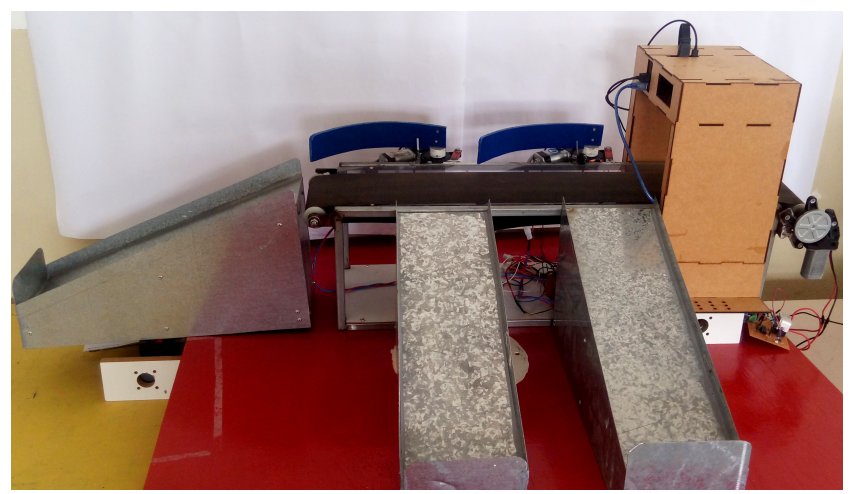

Figura 10. Sistema de classificação e seleção de mangas.

\section{CONCLUSÃO}

A realização deste trabalho resultou na construção de um sistema inteligente capaz de classificar e selecionar mangas em três estágios de maturação predefinidos utilizando redes neurais convolucionais aplicada a um processo de manufatura automatizado. A definição e implementação dos algoritmos de reconhecimento das mangas, através de imagens 2D (altura x largura) utilizando aprendizado profundo e mais especificamente uma rede neural convolucional, permitiu o emprego da própria imagem como entrada da rede, sendo a extração das características mais relevantes executada pelo algoritmo de rede ao longo dos filtros aplicados nas camadas de convolução.

O treinamento da rede com os dados obtidos pela câmera e em pesquisa online e ainda aplicando técnicas de aumento de dados, com a inversão horizontal e vertical da imagem, permitiu o ajuste de pesos do algoritmo de classificação para a realização das predições e uma probabilidade inerente a mesma. Os testes executados permitiram a validação do aprendizado da rede e ainda a definição da melhor configuração de mini-lote e número de passos para o desempenho considerando a acurácia e a perda no processo de predição. O maior desempenho do modelo de classificação foi exibido na predição das mangas verdes assim como a melhor distribuição de probabilidade.

A implementação do sistema de seleção permitiu o direcionamento adequado do mesmo de acordo com o estágio de maturação. O mecanismo empregado nesse processo foi capaz de efetuar a seleção das frutas de forma correta. Os testes realizados através da implementação do modelo treinado no sistema possibilitaram a definição da altura adequada da câmera em relação a esteira, a análise da intervenção do sistema de iluminação e interferência da iluminação do próprio ambiente em que se encontra o sistema e ainda a influência da resolução utilizada para captura da imagem, sobre o qual conclui-se que o aplicação de diferentes resoluções resulta na perda da distribuição de probabilidade da predição rede. A comunicação serial entre os subsistemas se mostrou eficiente e os atuadores foram acionados conforme previsto no projeto.

\section{AGRADECIMENTOS}

Ao IFBA e ao Grupo de Inovação e Pesquisa em Automação e Robótica (GIPAR) pelo suporte no desenvolvimento do trabalho.

\section{REFERÊNCIAS}

Bargoti, S. and Underwood, J. (2017). Deep fruit detection in orchards. In IEEE. 2017, International Conference on Robotics and Automation (ICRA), pages 3626-3633, 2017.

Barth, R., Ijsselmuiden, J., Hemming, J. and Henten, E. V. (2018). Data syn- thesis methods for semantic segmentation in agriculture: A capsicum annuum dataset. Computers and Electronics in Agriculture, pages 284$-296,2018$.

Brasil. (2017). Mais de 67\% das lavouras usam tecnologia na produção agrícola: Instrumentos tecnológicos apoiam desenvolvimento da agricultura de precisão. Disponível em: <http://www.brasil.gov.br/noticias/infraestrutura/201 7/04/mais-de-67-das-lavouras-usam-tecnologia-naproducao-agricola $>$. Acesso em: 09 de jan. de 2019.

Carvalho, J. N., Gurjão, E. C., Mata, M. E. R. M. C. e Duarte, M. E. M. (2014). Classificação Automática de Frutas por Análise de Imagem: O caso da Manga 
Tommy Atkins. In WTA. 2014, Workshop de Tecnologia Adaptativa, 8th edition, 2014.

Comércio Exterior - COMEX. (2017). Exportação: nos últimos três anos, a manga é a fruta que gera maior receita para o Brasil. Disponível em: <https://www.comexdobrasil.com/exportacao-nosultimos-tres-anos-manga-e-fruta-que-gera-maiorreceita-para-o-brasil/ $>$. Acesso em: 10 de jan. de 2019.

Embrapa Brasileira de Pesquisa Agropecuária - EMBRAPA. (2000). Manga: Pós-colheita. Embrapa Comunicação para Transferência de Tecnologia, Brasília, 2000.

Lecun, Y., Bottou, L., Bengio, Y. and Haffner, P. Gradientbased learning applied to document recognition. In Haykin, S. and Kosko, B. Intelligent Signal Processing. IEEE Press, pages 306-351, 2001.

Muresan, H. and Oltean, M. (2018). Fruit recognition from images using deep learning. Acta Univ. Sapientiae, Informatica, volume 10, 1st edition, pages 26-42, 2018.

Puttemans, S., Vanbrabant, Y., Tits, L. and Goedem, T. Automated visual fruit detection for harvest estimation and robotic harvesting. In IEEE. 2016, International Conference on Image Processing Theory, Tools and Applications (IPTA), pages 1--6, 2016.

Sa, I., Ge, Z., Dayoub, F., Upcroft, B., Perez, T. and Mccool, C. (2016). DeepFruits: A Fruit Detection System Using Deep Neural Networks. Sensors, volume 16, doi: $10.3390 / \mathrm{s} 16081222$.

Silva, F. S., Gottschall, C. R. A., Lima, D. S., Marques, J. E. S. and Amado, J. A. D. (2017). Desenvolvimento de um Controle Inteligente para Seleção de Mangas Aplicada a um Protótipo de Manufatura Robotizada. In MNR. 2017, Mostra Nacional de Robótica 2017, Anais, pages 703-707, 2017. 